\title{
Decision Support System for the Practical Implementation of the Chronic Heart Failure Guidelines: The MyHeart Approach
}

\author{
C Bescos ${ }^{1}$, M Harris ${ }^{1}$, R Bover ${ }^{2}$, R Schmidt ${ }^{1}, \mathrm{~J}$ Perez-Villacastin ${ }^{2}$ \\ ${ }^{1}$ Philips Research Laboratories, Aachen, Germany \\ ${ }^{2}$ Hospital Clinico San Carlos, Madrid, Spain
}

\begin{abstract}
Chronic Heart Failure (CHF) is the major cause for hospitalization in adults in western societies, mainly due to decompensation of patients. Active prevention, diagnosis, and personalized treatment contribute to the stabilization of chronic patients and the reduction of events. The approach of the EU FP6 MyHeart project is to collect daily vital body signs on CHF patients in an easy and comfortable way. The data is processed via a decision support system (DSS) and the platform gives instant recommendations to the user. The system also sends the information to the professionals for a better follow-up.

The designed DSS is based on Bayesian networks (BN) and combines the accepted standardized clinical guidelines with the most advanced monitoring data in daily routine, in order to provide individualized recommendations to the patient in a concrete situation.
\end{abstract}

\section{Introduction}

Despite the improvements in the treatment of Chronic Heart Failure (CHF), the prevalence in western countries is growing ( $4 \%$ of the adult population in Europe) and is likely to increase due to its link with age and other cardiovascular diseases. Treatment of CHF is a considerable economic burden. The direct costs of HF management are between 1 and $2 \%$ of the total healthcare budget [1].

As the new guidelines in management of CHF show, more active prevention, diagnosis and personalized treatment need to be established [2]. Translating the benefits of the evidenced based therapy to the routine of the HF population has proven to be costly. Patients with CHF find it difficult to comply with the assigned regimes due to their age, associated additional comorbidities, and/or to the complexity of the therapy [3].
A systematic review of literature on the effect of health ICT on quality, efficiency and costs of care found that three major benefits on quality were demonstrated: increased adherence to guideline-based care, enhanced surveillance and monitoring, and decreased medication errors [4]. Concretely, in the area of chronic diseases, by closely monitoring and evaluating certain clinical parameters in chronic patients, it has been shown in small trials that it is possible to predict decompensation of these patients before symptoms of deterioration occur (and the combined end-point of rehospitalisation rate and mortality was reduced) [5].

MyHeart is an Integrated Project of the European Union aimed at developing intelligent systems for the prevention and monitoring of cardiovascular status. The approach of the MyHeart project is to monitor Vital Body Signs with wearable and non-invasive technology, to process the measured data and to give the user (therapy) recommendations from the system. Using MyHeart's broad base of technical and business expertise, four concepts addressing cardiac health have been developed and tested on a technical, business, realisability and usability level [6].

This paper is based on the results from the MyHeart Heart Failure (HF) Management concept, whose main objective is to improve the outcome of chronic heart failure patients with respect to mortality, morbidity and quality of life. The platform monitors remotely vital body signs that are relevant for heart failure on a daily basis (currently these parameters are only measured at less frequent visits to the physician). The system is designed to detect changes in the patient's health status early enough to allow the timely therapy intervention from the professional, thus avoiding severe deterioration and hospitalisation. The end users of the system are patients with heart failure (NYHA classes II-IV), and the physicians and nurses caring for the patient.

An observational study of one year of follo-up with 200 heart failure patients will be carried out in Germany and Spain [7]. The MyHeart HF system will be used to 
make daily measurements of vital body parameters. At the end of the multi-centre study the medical incidents will be correlated with the measured data to deduce which parameters can be used, in which combination, to give warnings of a forthcoming decompensation.

The Decision Support System (DSS) explained in this paper represents the initial prototype to assist patients and professionals in the interpretation of vital body signs evolution for the better follow-up of HF patients. The MyHeart HF Observational Study will provide the necessary clinical evidence of the parameters considered in the DSS system.

\section{Methods}

The DSS added-value is the ability of providing clinicians, patients or individuals with knowledge and person-specific or population information, intelligently filtered or presented at appropriate times, to foster better health processes, better individual patient care, and better population health. Hence, a DSS does not mean replacing the role of the professionals, but to assist both patients and clinical staff in receiving and applying the best personalized treatment, while optimizing the resources.

The system is designed according to the methodology for Bayesian Networks (BN) life cycle [8] showed in figure 1 below. The context creation, the selection of variables and ranges has been performed with the collaboration of cardiologists. The model needed some initial estimation from the experts, and then the BN learns from the experience and adapts itself to the concrete cases. The platform is able to integrate the knowledge from the guidelines for $\mathrm{CHF}$, and furthermore it can incorporate readings from innovative sensors generating new reaction strategies based on past measurements and outcomes. The system thus learns from past cases, giving evidence based recommendations for subsequent cases.

$\mathrm{BN}$ are interesting for medical reasoning problems because:

- BN allow both top-down and bottom-up reasoning

- They facilitate decision under uncertainty

- They easily capture the experts reasoning in causeeffect terms.

- There are statistical tools and software available

- They can learn from data. They are easily updatable and personalisable

- There is extensive experience in application of BN in different medical solutions.

On the other hand, BN show some limitations:

- They do not provide a reasoning/explanation engine themselves.

- To solve a particular situation it is always needed to solve the complete network.

- They need a lot of data/knowledge to set the adequate probability distributions.

- They only consider mainly discrete/nonoverlapping variables.

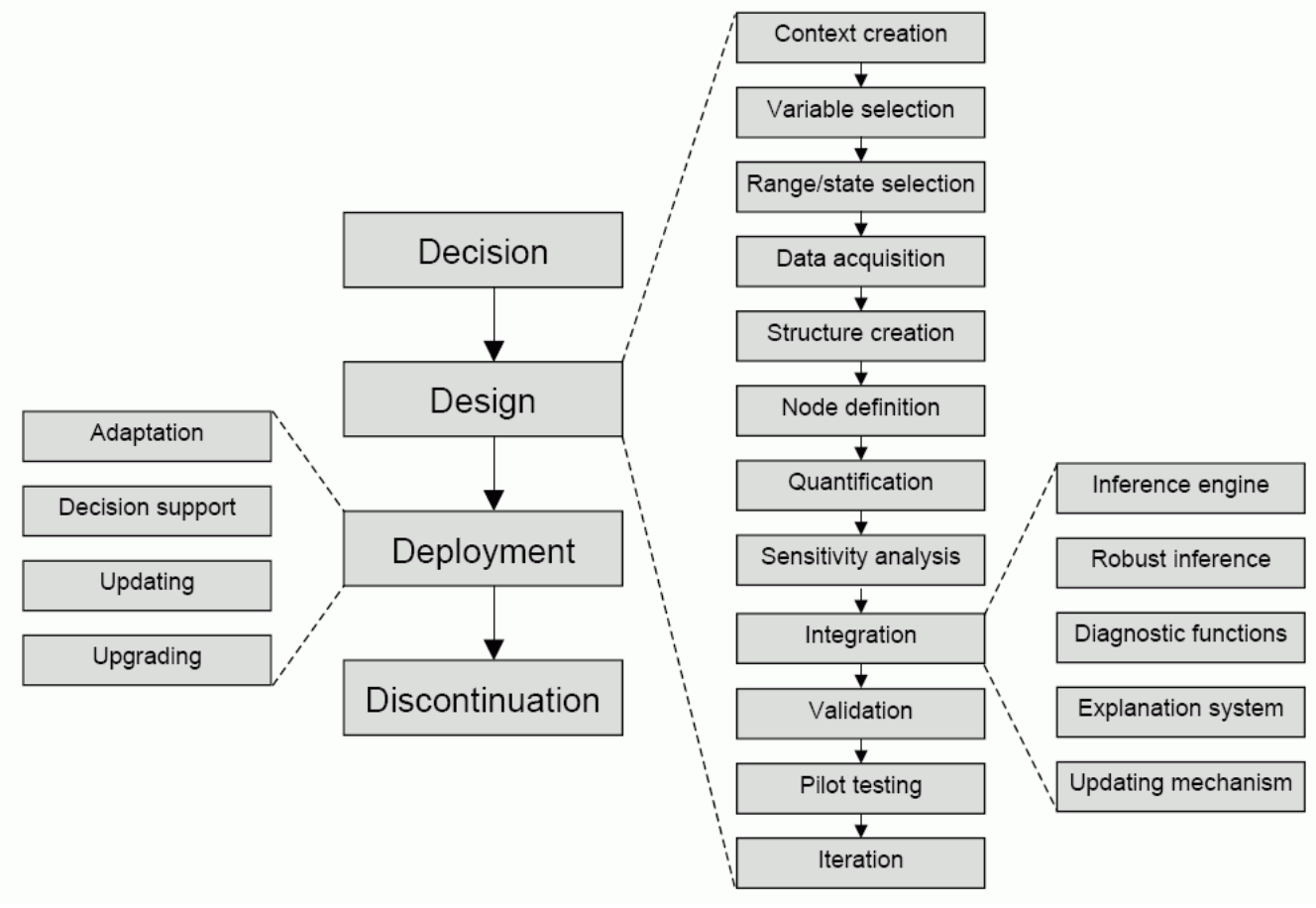

Fig.1: Bayesian Network Life Cycle [8] 


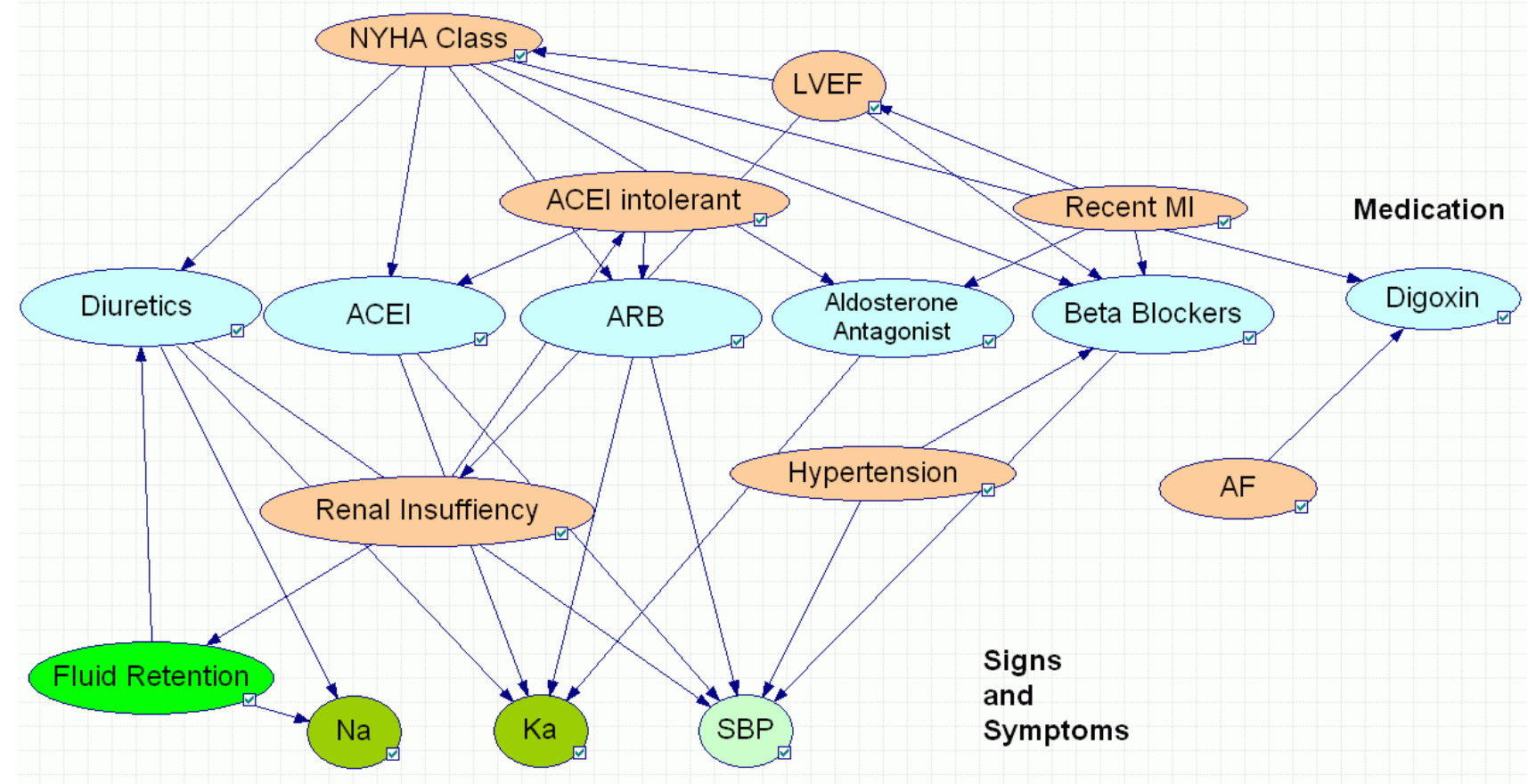

Fig 2.: Simplified structure of the Bayesian Network

\section{Results}

An initial prototype for the Decision Support System for Chronic Heart Failure has been developed and tested.

Figure 2 presents a simplified structure of the BN. The complete network consists of 1029 expanded nodes (486 target parameters, 523 observation and 20 auxiliary variables). The main input parameters are: New York Heart Association Class (NYHA), congestion symptoms, Left Ventricular Ejection Fraction (LVEF), recent history of myocardial infarction, tolerance to the Angiotensin Conversion Enzyme Inhibitors (ACE Inhibitors), diuretics, previous hypertension, systolic blood pressure, renal insufficiency and the Potassium and Sodium levels.

The influence of the initial parameters (introduced in the system by the medical team) determines the selection of the optimal medication: in moderate HF, the LVEF and the intolerance to the ACE Inhibitors are the most relevant variables. In the case of severe HF, the influence of secondary effects of the drugs is higher.

The BN has already been tested against the latest version (2005) of the European Society of Cardiology (ESC) guidelines, providing the recommended medication in the proposed use cases. A validation with cardiologists for the selection and quantification of the input and output variables has been completed.

Data from the MyHeart HF study mentioned in the introduction will be used to prove the efficacy in the prediction of the output values (evolution prognosis, clinical status during follow-up and decompensation risk) when there is uncertainty or no knowledge of one or some of the input values and will verify the system propagation of the uncertainty in the results.

Figure 3 shows the user interface for the professionals. First the system requests some initial parameters to calculate the recommended medication according, to European guidelines. The platform provides accurate guidance in the selection of the medication dosage, the incompatibilities of drugs and the titration periods and doses. Finally, the system has an additional functionality for the daily follow-up of patients. When integrated in the MyHeart platform, the solution will receive data from the patient sensors and will calculate the status and expected clinical evolution.

\section{Discussion and conclusions}

The developed DSS provides practical assistance to the professionals and non-specialists in the optimal management of CHF patients. The system does not try to substitute the clinical guidelines, but to foster and optimise its implementation for concrete patients.

In the past, some efforts in DSS in clinical settings have already shown potential, but mainly in the professional side. The key element of the presented DSS is a closed loop approach, which does not only monitor but also provides solutions for the patient at home, putting them at the centre of the system. 


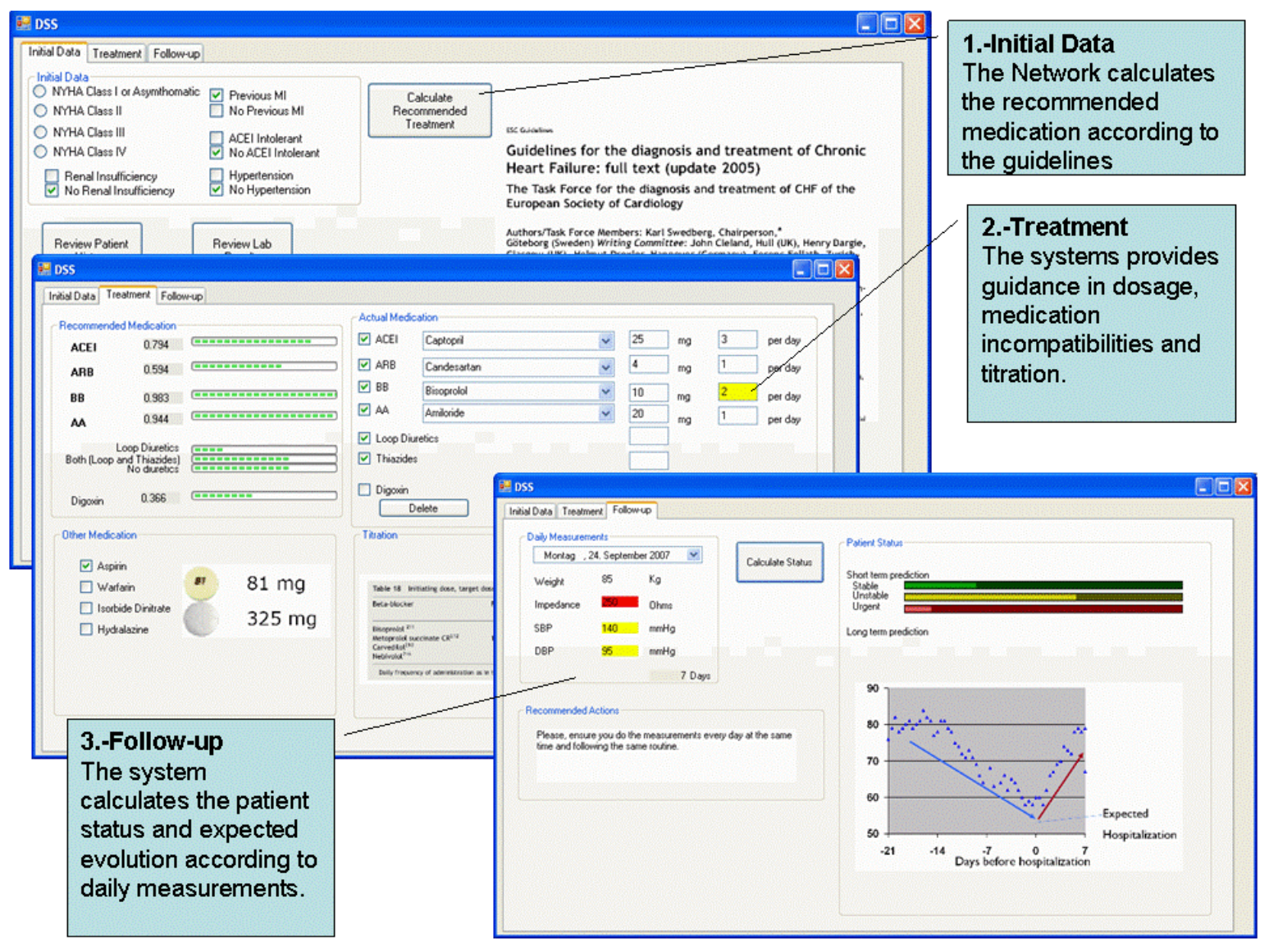

Fig. 3: DSS doctor user interface

The MyHeart HF Clinical Study will provide the necessary evidence for the validation of the quantification and personalization of the $\mathrm{BN}$. At that stage a sensitivity analysis will be performed following the $\mathrm{BN}$ life cycle shown in figure 1.

\section{Acknowledgements}

This work was supported by the European Marie Curie Transfer of Knowledge Program. Project AIMed, Advanced Intelligent Medication - personalized drug dosage concepts to improve quality of life. [TOK-14459].

The research was based on the results from the MyHeart Project [IST-2002-507816].

\section{References}

[1] Berry, C, Murdoch, DR, McMurray, JJV. Economics of chronic heart failure. European Journal of Heart Failure 2001:3:283-291.

[2] Guidelines for the diagnosis and treatment of chronic heart failure: executive summary (update 2005): The Task Force for the Diagnosis and Treatment of Chronic Heart Failure of the European Society of Cardiology. Eur Heart J 2005;26:1115-40.
[3] WHO report 2003: Adherence to long-term therapies: evidence for action.

[4] American Medical Informatics Association. "A Roadmap for National Action on Clinical Decision Support". 2006

[5] Cleland, J. G. F., Louis, A. A., Rigby, A. S., Janssens, U., Balk, A. H. M. M., on behalf of the TEN-HMS investigators, 'Noninvasive home tele-monitoring for patients with heart failure at high risk of recurrent admission and death: The Trans-European Network-HomeCare Management System (TEN-HMS) Study', J Am Coll Cardiol,vol. 45(10), pp. 1654-1664, 2005.

[6] MyHeart IST-2002-507816 project (2004). Information available on http://www.cordis.lu/ist

[7] MyHeart. Prediction of Decompensation in Heart Failure patients. Observational Study. Investigational plan. 2007

[8] van Zon, K. The Bayesian Network Lifecycle. Technical Note PR-TN-2006/00155. Export review: EAR99 NLR. Philips Research North America, NY, USA 2006

Address for correspondence

Cristina Bescos

Philips Research Labs

Weisshausstrasse 2,

52066 Aachen,

Germany.

Cristina.Bescos@philips.com 